\title{
Application d'une méthode d'étude quantitative et qualitative des rejets liquides hospitaliers au niveau de la Région de Marrakech Tensift El Haouz, Maroc
}

\author{
Qadouri Asmaa \\ Mouhir Latifa
}

Laboratoire Génie de l'Eau et de l'Environnement, Faculté des Sciences et Techniques de Mohammedia, (Maroc)

\section{Belkadi Mohammed Said}

Centre Hospitalier Mohammed VI, Marrakech (Maroc)

doi: 10.19044/esj.2016.v12n32p110 URL:http://dx.doi.org/10.19044/esj.2016.v12n32p110

\begin{abstract}
This study led to the development of a decision support tool to quantify the hospital effluent generated by public and private health care establishments in the region of Marrakech-Tensift-El Haouz situated in Morocco. The estimation concerns the consumption in drinking water, the production in wastewater and on the most consummate products collectively in the whole of the establishments of care and which join the sewer system (cleaner, soap, washing, Glutaraldehyde) based on the capacity litter, the hospitable vocations and the production rate. By having the results of quantification of the deposit rejected in the municipal network, the present study estimates then for the case of the city of Marrakesh the part of contribution of the hospital effluent in the municipal pollution.
\end{abstract}

Keywords: Liquid medical and pharmaceutical waste, establishment of care, public, private, region of Marrakech-Tensift-El Haouz

\section{Résumé}

L'étude a développé un outil d'aide à la décision pour la quantification des rejets liquides des établissements de soin publics et privés au niveau de la région de Marrakech Tensift El Haouz-Maroc. L'estimation porte sur la consommation en eau potable, la production en eau usée et sur les produits les plus consommés communément dans l'ensemble des établissements de soin et qui rejoignent le réseau d'assainissement (détergent, savon, lessive, glutaraldhéhyde) en se basant sur la capacité litière, les vocations hospitalières et les ratios de production. En ayant les 
résultats de quantification du gisement rejeté dans le réseau communal, ce travail évalue ensuite pour le cas de la ville de Marrakech la part de contribution des rejets des hôpitaux dans la pollution communale.

Mots clefs : Déchets médicaux et pharmaceutiques liquides, établissement de soin, public, privé, région de Marrakech-Tensift -El Haouz

\section{Introduction}

$\mathrm{Au}$ Maroc, la problématique des déchets médicaux et pharmaceutiques liquides (DMPL) n'a pas pris encore l'ordre des priorités auprès des décideurs pour être traiter. Aujourd'hui, aucune obligation n'incombe les producteurs des DMPL sur les modalités de gestion et les processus de traitement spécifiques de ces rejets. Seuls les rejets issus des services d'oncologie font objet d'une gestion appropriée de la production jusqu'au traitement, le reste des rejets liquides rejoint le réseau des eaux usées domestique même si le risque et l'impact des effluents hospitaliers sur l'homme et l'environnement est prouvé dans plusieurs travaux de recherche. Encore moins, aucune norme marocaine concernant les résidus pharmaceutiques ou pathogènes dans les effluents hospitaliers n'est promulguée.

Les eaux usées hospitalières peuvent représenter un danger pour la santé humaine, en particulier à l'intérieur de l'hôpital, le personnel soignant et technique, les patients, les visiteurs, les employés des stations d'épuration.

Une fois les effluents relâchés dans l'environnement avec leur part résiduelle d'agents pathogènes et de produits pharmaceutiques, la faune et la flore locale est constamment en danger.

La spécificité des rejets liquides hospitaliers est dans le degré de la toxicité. Même à très faible concentration, les substances contenues dans les eaux usées provenant d'un hôpital peuvent affecter la vie animale; Les œstrogènes, par exemple, peuvent causer l'apparition de poissons hermaphrodites. Certains antalgiques sont toxiques pour les truites et certains médicaments psychoactifs peuvent modifier le comportement des poissons et des oiseaux. [1]

Pour faire face à la question des eaux usées hospitalières, l'objectif de cette étude est de quantifier le gisement rejeté afin de trouver une solution de gestion et de traitement permettant d'éliminer les substances problématiques dans les effluents hospitaliers plutôt que de les diluer avec d'autres effluents dans le réseau public d'assainissement.

Et d'autre part, une attention croissante récemment a été portée à la réutilisation des eaux usées traitées en irrigation au Maroc vu que la demande d'eau potable ne pouvant pas être totalement satisfaite par des eaux 
souterraines, d'autres sources, telles que les eaux de surface, ont dû être considérées.

Le présent travail présente un outil d'aide à la décision pour quantifier les rejets liquides hospitaliers et les produits liquides les plus consommés communément dans les établissements de soin publics et privés. La région étudiée est la région de Marrakech Tensift El Haouz avec une étude de cas détaillée de la ville de Marrakech.

\section{Matériels et méthodes}

\section{Zone d'étude}

La zone d'étude concerne la région de Marrakech Tensift El Haouz.

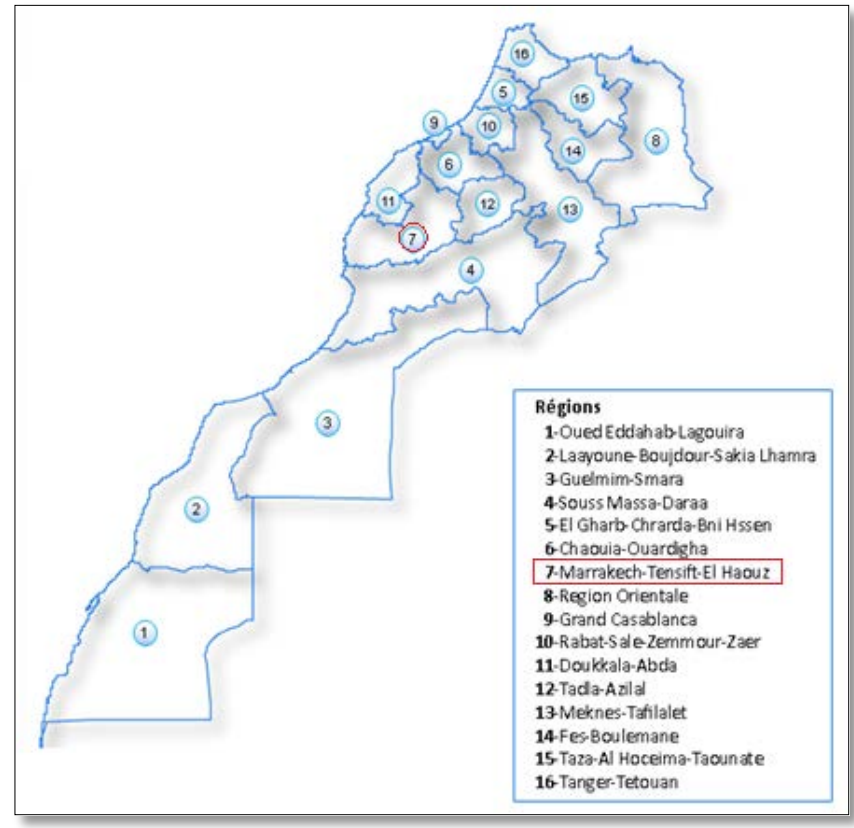

Carte $\mathrm{n}^{\circ}$ 1: Provinces du Maroc

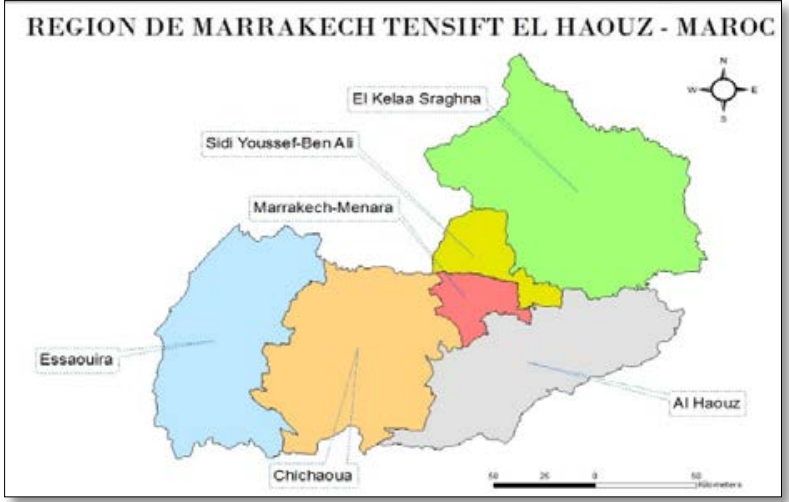

Carte $\mathrm{n}^{\circ} 2$ : Provinces de la région de Marrakech-Tensift-El Haouz 


\section{Matériels}

Statistiques du Ministère de santé 2013, édition 2014

Les données sur la capacité litière les spécialités des établissements de soin publics et privés réparties dans les provinces de la région de Marrakech Tensift El Haouz [2] sont exploitées dans la présente étude.

\section{Logiciels}

Le logiciel utilisé pour la présentation graphique est le module analyste spatiale du logiciel ArcGis $10 \AA$. Le traitement statistique est réalisé par Excel.

\section{Méthodes}

La méthode d'estimation appliquée est un outil simple qui facilite l'évaluation de la gestion des rejets liquides au sein des établissements de soin sur le plan qualitatif et quantitatif. Cette méthode consiste à calculer la quantité produite des liquides les plus consommés communément dans les établissements de soin et retournés à l'égout. La production de ces liquides est liée à la taille de l'établissement de soin (capacité litière), la vocation hospitalière et les ratios de production retenus à l'échelle internationale [3].

\section{Taux d'occupation des lits d'hôpitaux}

Selon [4], Le taux d'occupation des lits d'hôpitaux représente la somme des journées d'hospitalisation au cours d'une période donnée (en général une année) divisée par le nombre de lits disponibles, Il indique le degré d'utilisation des lits disponibles durant une période déterminée

Selon les chiffres du Ministère de santé [5], à l'échelle nationale en 2012 les établissements publics ont un taux d'occupation moyen (TOM) de $62.6 \%$.

Le taux d'occupation moyen adopté pour le secteur public est de $60 \%$ et pour le secteur privé est de 50\%. Cela pour une meilleure représentativité de l'ensemble des établissements de soin au niveau de la région étudiée.

\section{Consommation en eau potable dans les établissements de soin} facteurs :

La consommation en eau potable varie en fonction de plusieurs

- Taille de l'établissement: CHU, CHR, clinique privé/public, cabinet,

- Vocation de l'établissement: psychiatrique, chirurgicale, oncologique, soins de base...;

- Pratique et fréquence d'hygiène ;

- Etc. 
Pour notre étude, la valeur moyenne adoptée est de $200 \mathrm{~L} / \mathrm{lit} / \mathrm{jour}$ pour une représentativité des différents établissements de soin au niveau des villes et au niveau de la région étudiée. Par ailleurs, une étude ponctuelle d'un établissement de soin doit quantifiée d'une façon plus succincte les consommations en eau par service.

\section{Production en eau usée}

Au Maroc, le taux de retour à l'égout adopté dans les études d'assainissement liquide est de $80 \%$ des eaux consommées pour les eaux d'origine domestique et administrative selon le Schéma Directeur National d'Assainissement Liquide [6].

L'estimation de la production en eau usée à l'échelle régionale dans la présente étude tient en considération uniquement des consommations en eau potable et les ratios de rejet pour chaque produit liquide étudié. Pour une étude ponctuelle de la quantification des rejets liquides au niveau d'un établissement de soin il faut prendre en compte tous les inputs en matière d'eau consommée à savoir les eaux importées de l'extérieur (eau physiologique, eau des puits,...).

\section{Choix des produits les plus consommés Produits}

Aujourd'hui, il existe une large fourchette de produits liquides dans le marché. Et selon les besoins de chaque spécialité de médecine, l’hôpital utilise d'une manière continue une panoplie de produits liquides et solides solubles dans l'eau avec des quantités variables et indéterminées. Ainsi pour notre étude, les produits faisant objet de choix sont des liquides utilisés d'une façon commune dans l'ensemble des établissements de soin à savoir :

- Produits d'entretien des locaux ;

- Produits utilisés dans la blanchisserie : Lessive, eau consommée ;

- Produits de désinfection utilisés dans l'activité médicale : Savon doux ou antiseptique, gludaraldéhyde ;

- Détergent pour lave-vaisselle.

\section{Ratio de pollution}

Les chiffres clés pour quantifier les rejets liquides potentiellement à risque pour l'homme et l'environnement utilisés dans un établissement de santé de 1000 lits sont comme suite [7]. 
Tableau 1 : Quantités annelles des produits liquides les plus consommés dans les établissements de soin par activités [3]

\begin{tabular}{|c|c|c|c|}
\hline Activités & Produits & $\begin{array}{c}\text { Quantité } \\
\text { annuelle }\end{array}$ & Remarques \\
\hline $\begin{array}{c}\text { Entretien des } \\
\text { locaux }\end{array}$ & Détergent & 5 à $10 \mathrm{~m} 3$ & $\begin{array}{c}\text { Selon la structure de l'établissement : } \\
\text { concentré ou pavillonnaire }\end{array}$ \\
\hline \multirow{2}{*}{ Blanchisserie } & Eau & $\begin{array}{c}18000 \text { à } \\
26000 \mathrm{~m} 3\end{array}$ & Selon le procédé de lavage \\
\cline { 2 - 4 } & Lessive & $13 \mathrm{~T}$ & \\
\hline $\begin{array}{c}\text { Activités de } \\
\text { soins }\end{array}$ & Savon & 7 à $10 \mathrm{~m} 3$ & Doux ou antiseptique \\
\cline { 2 - 4 } & Glutaraldhéhyde & 2 à $4 \mathrm{~m} 3$ & Suivant l'activité chirurgicale \\
\hline Cuisine & Détergent & $4 \mathrm{~T}$ & Pour lave-vaisselle \\
\hline
\end{tabular}

Dans notre étude, les ratios adoptés sont les valeurs les plus basses pour chaque intervalle des quantités annuelles consommées citées dans le tableau $\mathrm{n}^{\circ} 1$ et ceux pour tenir compte d'une part de l'hétérogénéité des vocations médicales et leurs besoins en consommation et d'autre part pour tenir compte de la taille des établissements et le contexte urbain et rurale.

\section{Méthode de calcul}

En ayant la capacité litière, la vocation des établissements de soin privés et publics au Maroc [2] ainsi que les ratios de pollution pour les produits liquides les plus utilisés et rejetés dans les égouts des établissements de soin, la méthode d'estimation est basée sur la formule de calcul adopté suivante :

V (produit liquide étudié) $=$

Capacité litière de l'établissement de soin étudié* Ratio de consommation du produit liquide étudié

$$
1000
$$




\section{Résultats et discussion \\ Résultats \\ Cartographie de l'offre de soin publique détaillée dans la région de Marrakech Tensift El Haouz}

OFFRE DE SOIN PUBLIQUE DANS REGION DE MARRAKECH TENSIFT EL HAOUZ - MAROC

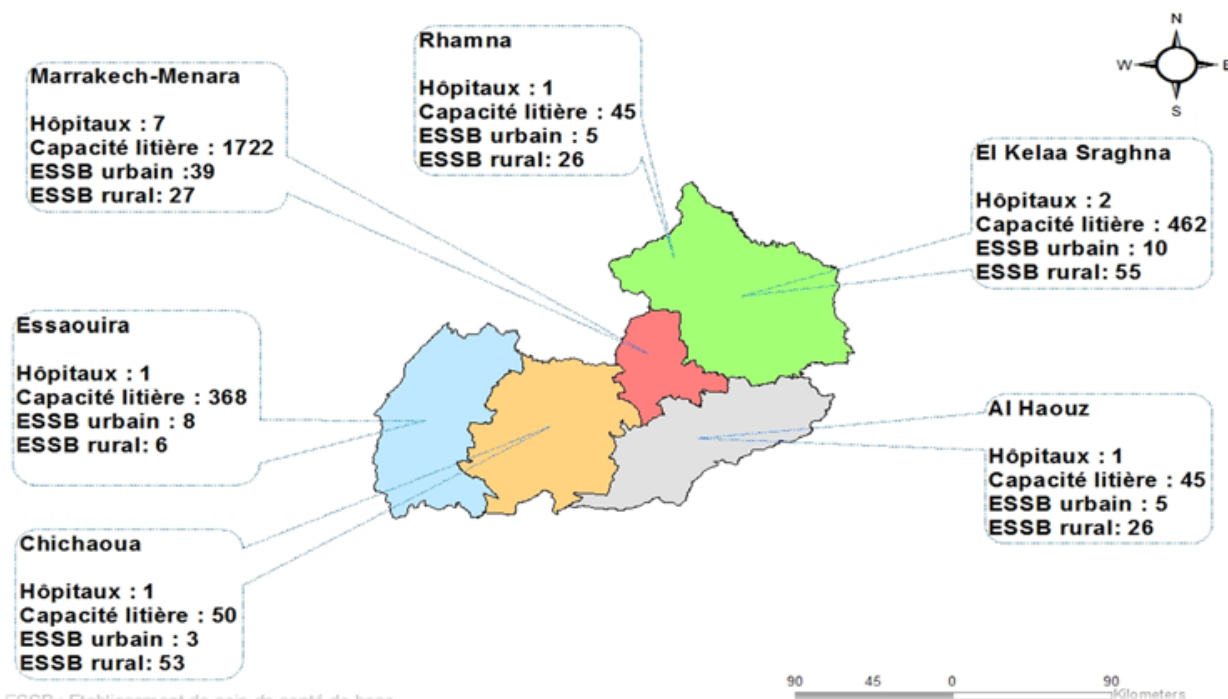

Carte $n^{\circ}$ 3: Offre de soin publique dans la région de Marrakech Tensift El Haouz, 2014

\section{Cartographie de l’offre de soin privée détaillée dans la région de Marrakech Tensift El Haouz}

OFFRE DE SOIN PRIVEE DANS REGION DE MARRAKECH TENSIFT EL HAOUZ - MAROC

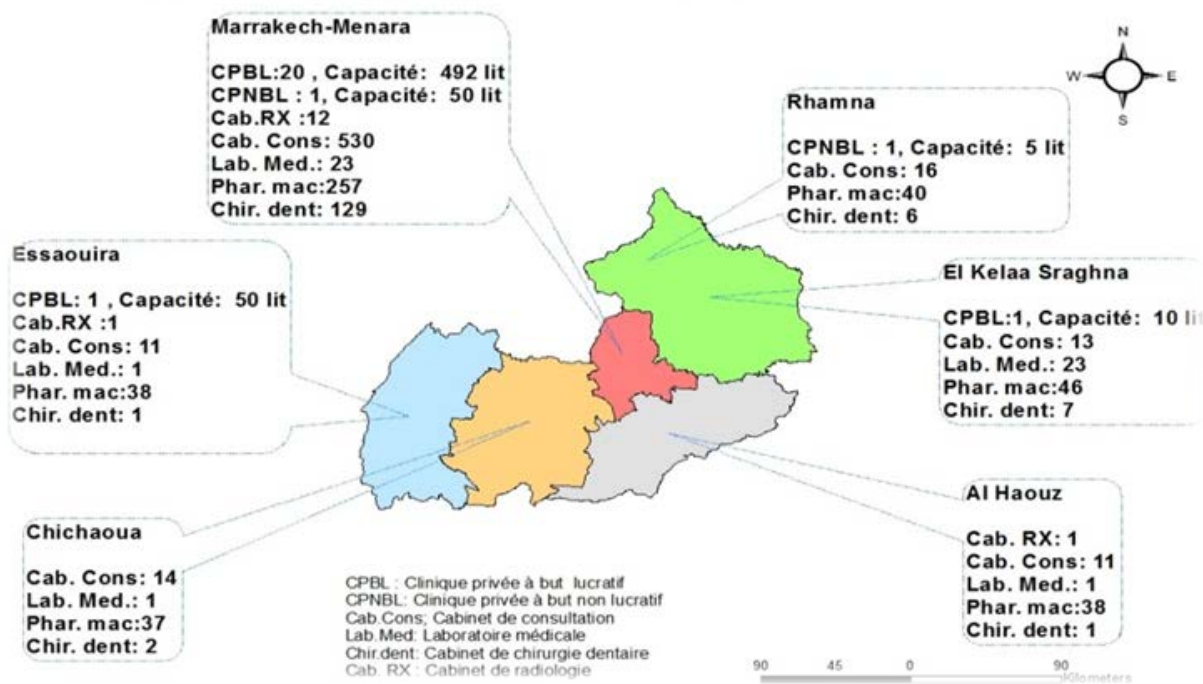

Carte $n^{\circ} 4$ : Offre de soin privée dans la région de Marrakech Tensift El Haouz, 2014 
Cas des établissements de soin publics dans la Région de Marrakech Tensift El Haouz

\section{a/ Capacité litière et Taux d'occupation moyen}

Capacité fonctionnelle dans les établissements de soin publics

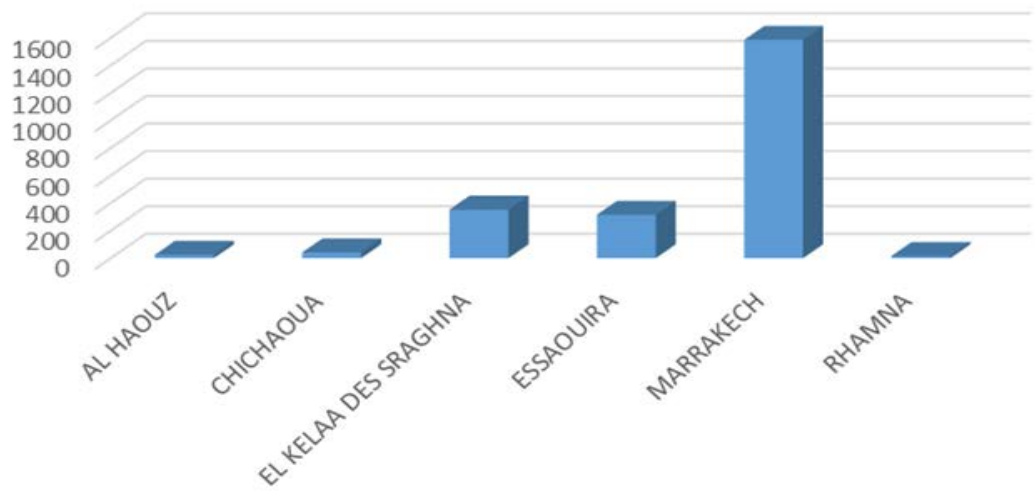

Taux d'occupation moyen

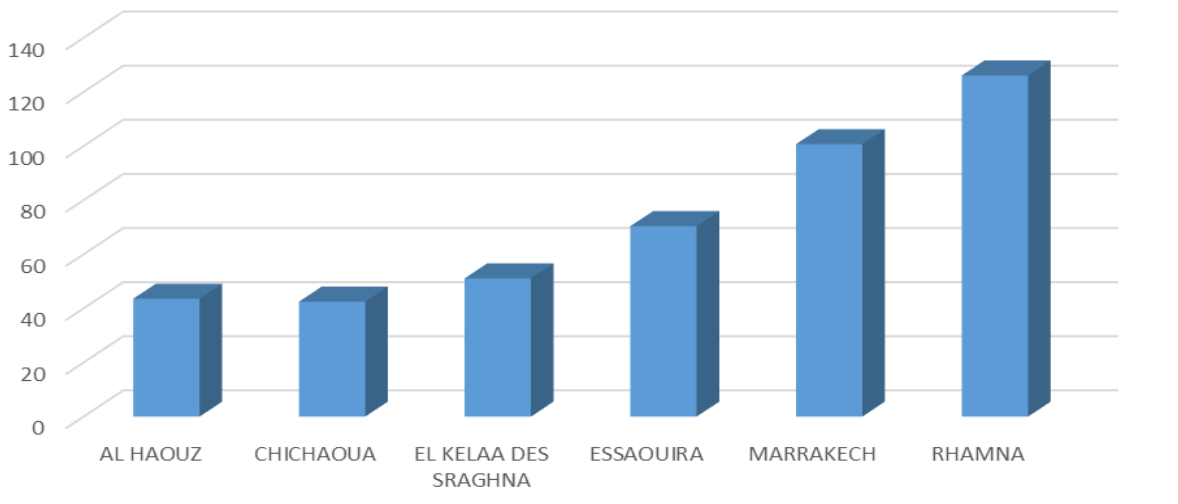


b/ Consommation en eau potable -Production en eau usée

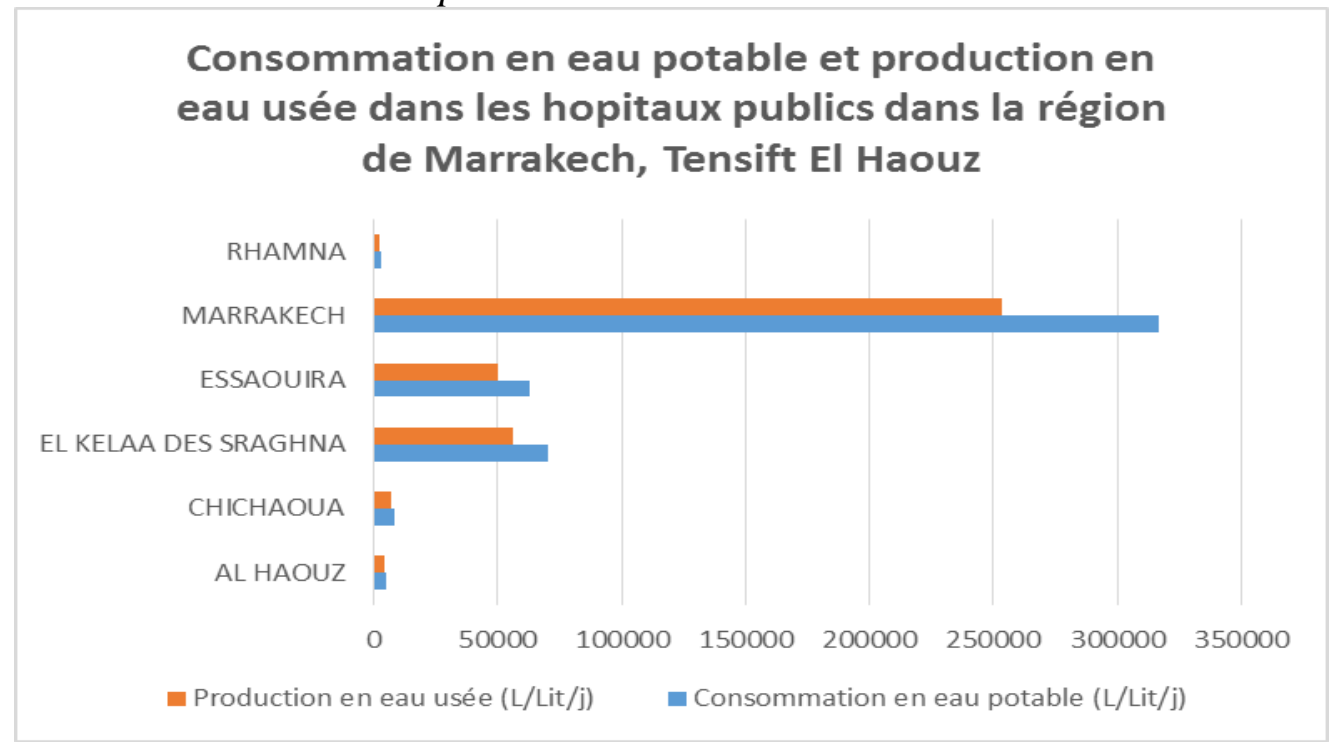

La consommation en eau potable et la production en eau usée sont proportionnelles à la capacité litière au niveau des établissements de soin pour le secteur privé et public.

Pour le cas des établissements de soins publics dans la région de Marrakech Tensift El Haouz, la ville de Marrakech est en tête avec une capacité litière fonctionnelle de 1585 lit selon [2], une consommation en eau potable qui dépasse les $300000 \mathrm{~L} / \mathrm{j}$ et une production en eau usée d'environ $250000 \mathrm{~L} / \mathrm{j}$. Pour les quatre communes restantes, elles ne dépassent pas chacune un seuil de $70000 \mathrm{~L} / \mathrm{j}$.

c/ Quantification des produits liquides les plus consommés

c-1 Révélateur et fixateur de l'imagerie médicale, détergents (entretien des locaux), savon et glutaraldéhyde (activité de soin) 


\section{Consommation des produits liquides les plus utilisés dans les hopitaux publics}

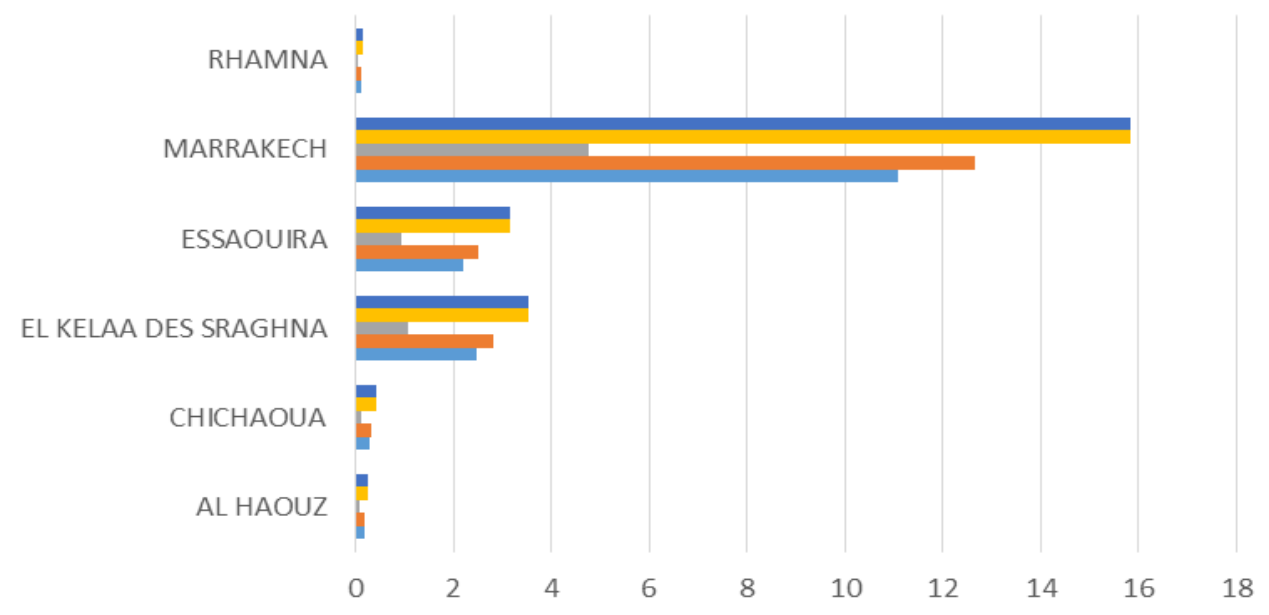

- Imagerie médicale Révélateur(m3/an) I Imagerie médicale Fixateur (m3/an)

- Activités de soins Glutaraldéhyde (m3/an) — Activités de soins Savon (m3/an)

- Entretien des locaux Détergent (m3/an)

c-2 Détergent (cuisine), lessive (blanchisserie)

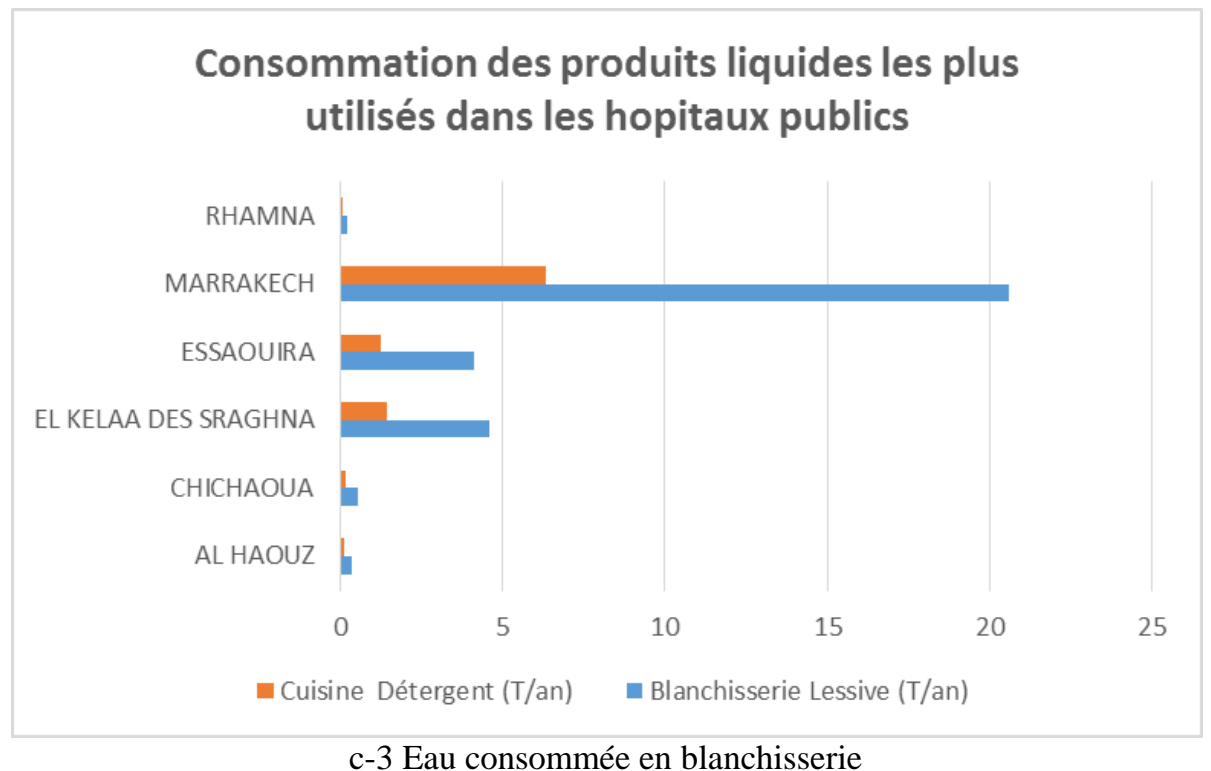

c-3 Eau consommée en blanchisserie 


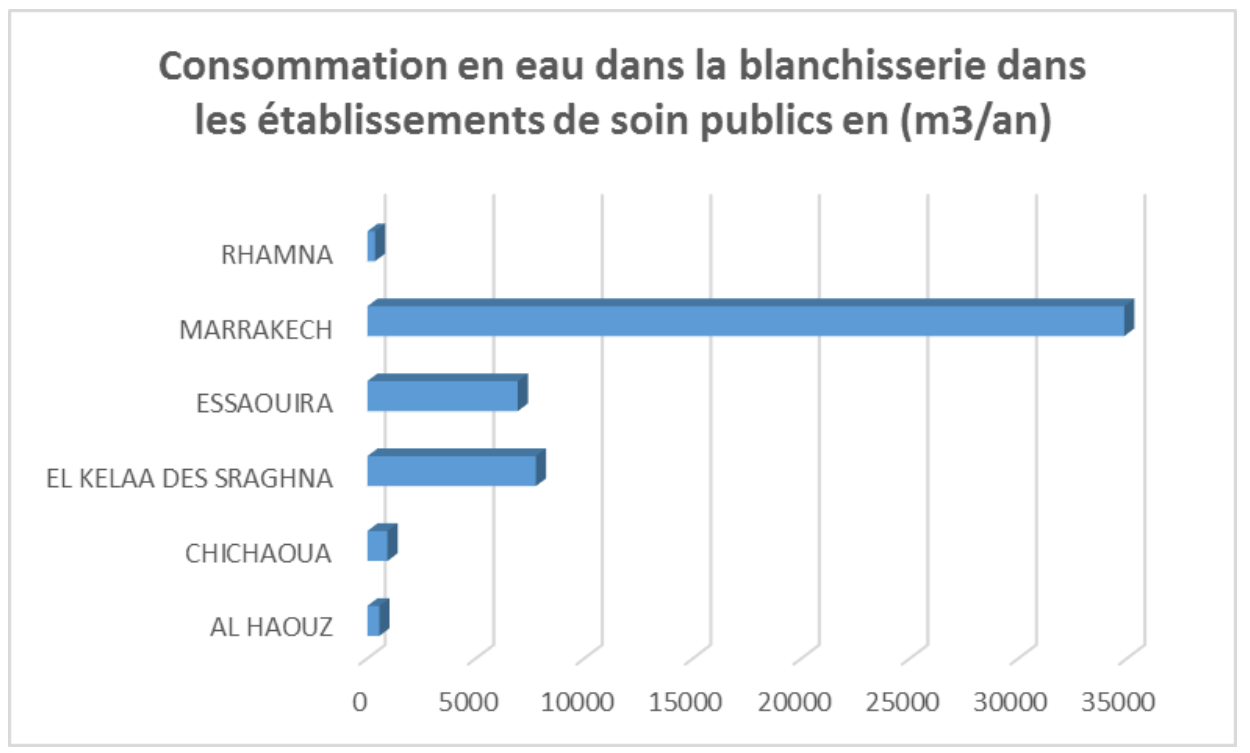

Cas des établissements de soin privés dans la Région de Marrakech Tensift El Haouz

a/ Capacité litière

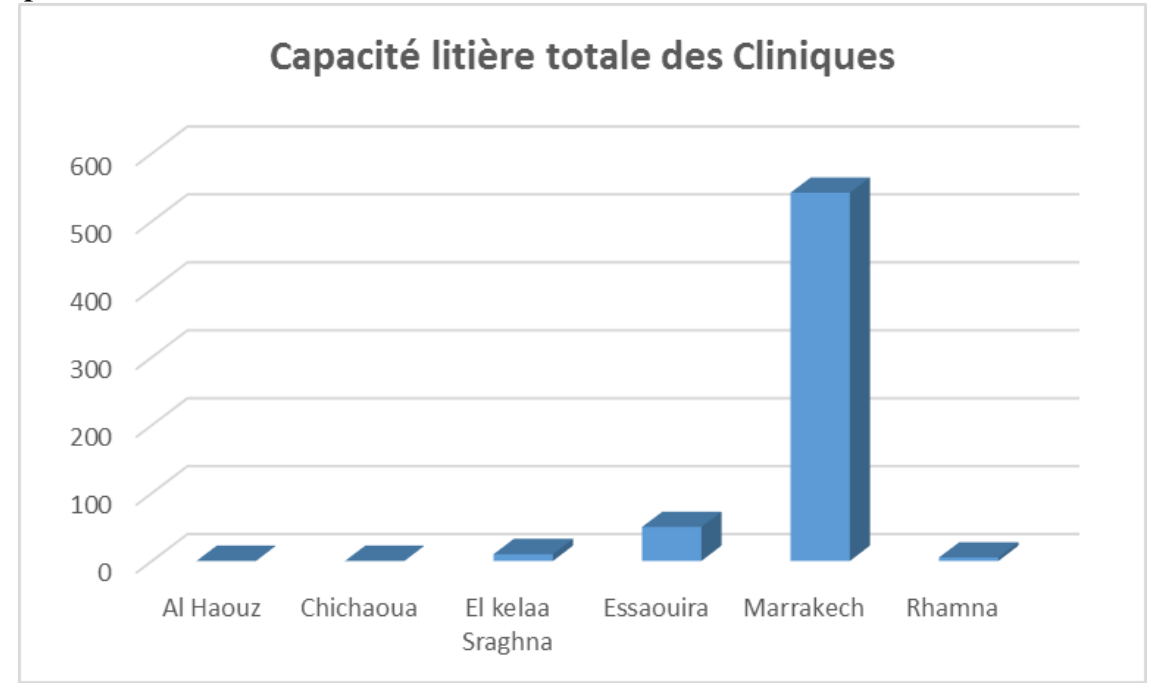

Le secteur présenté dans cette étude concerne les cliniques privées ayant une capacité litière fonctionnelle reportée dans [2]. 
b/Consommation en eau potable -Production en eau usée

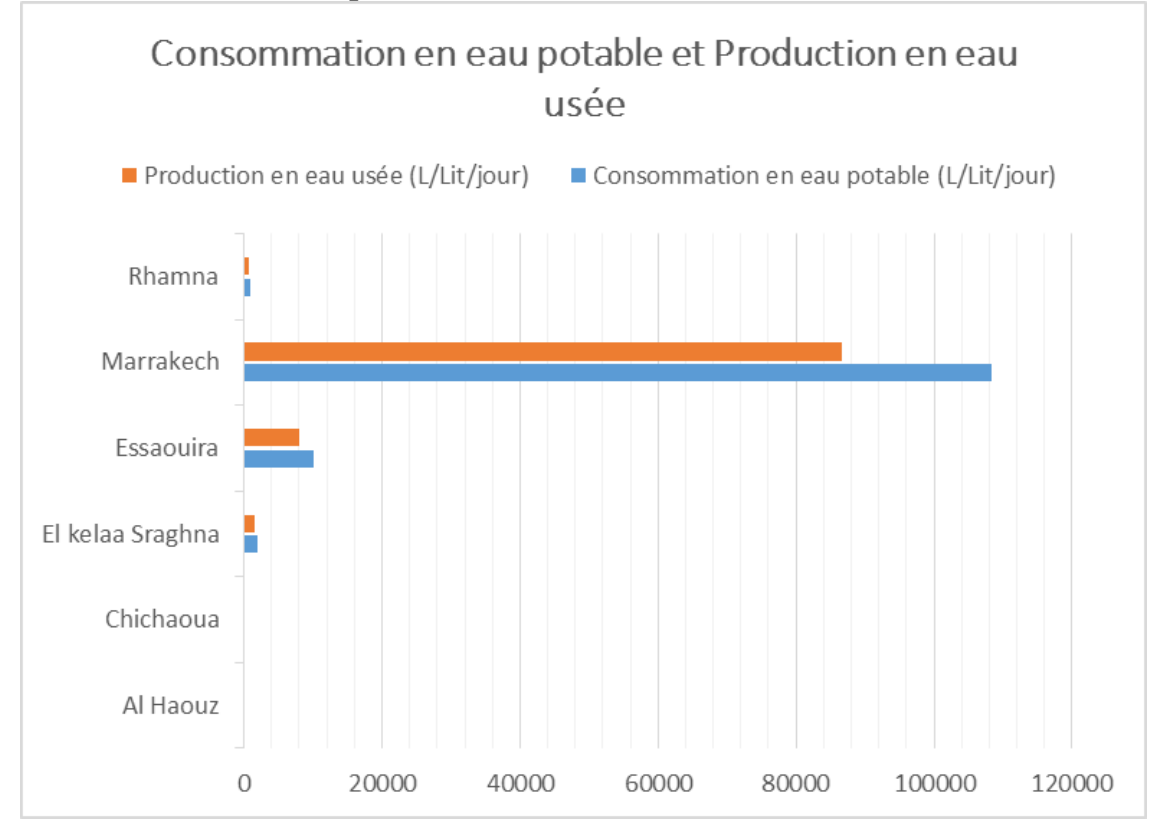

Comme pour le cas des établissements de soins privés dans la région de Marrakech Tensift El Haouz, la ville de Marrakech est en tête avec une capacité litière fonctionnelle de 542 lits en 2015, une consommation en eau potable d'environ $108000 \mathrm{~L} / \mathrm{j}$ et une production en eau usée de 86720 $\mathrm{L} / \mathrm{j}$. Pour les quatre communes restantes, elles ne dépassent pas chacune un seuil de $10000 \mathrm{~L} / \mathrm{j}$.

c/ Quantification des produits liquides les plus consommés

c-1 Eau consommée en blanchisserie

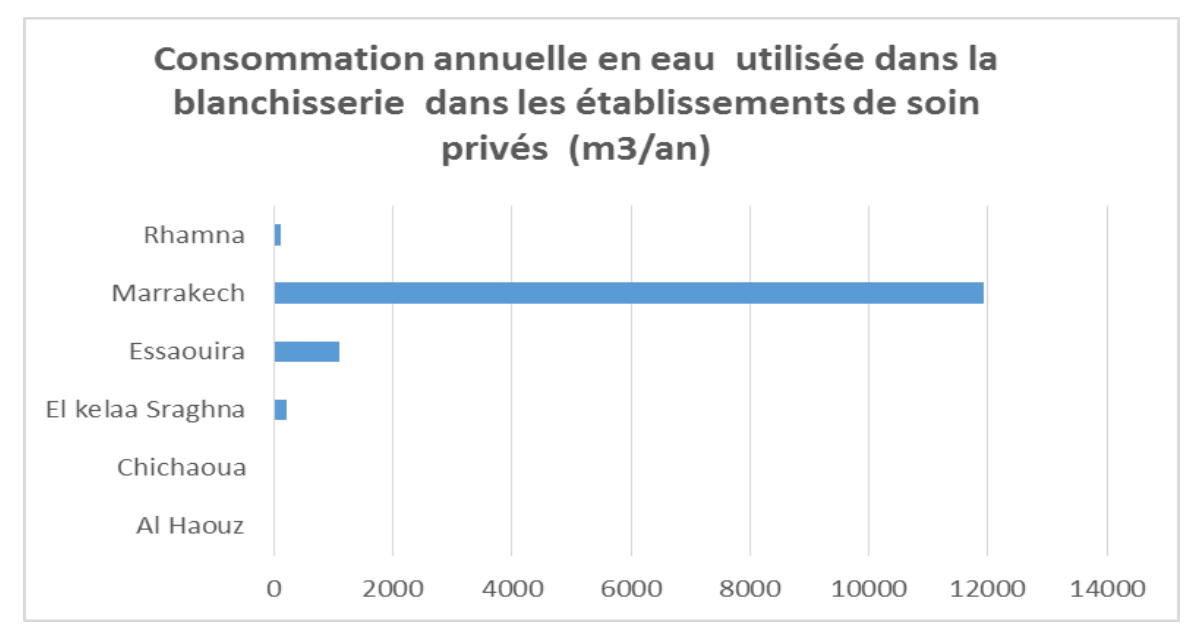


c-2 Révélateur et fixateur de l'imagerie médicale, détergents (entretien des locaux), savon et glutaraldéhyde (activité de soin)

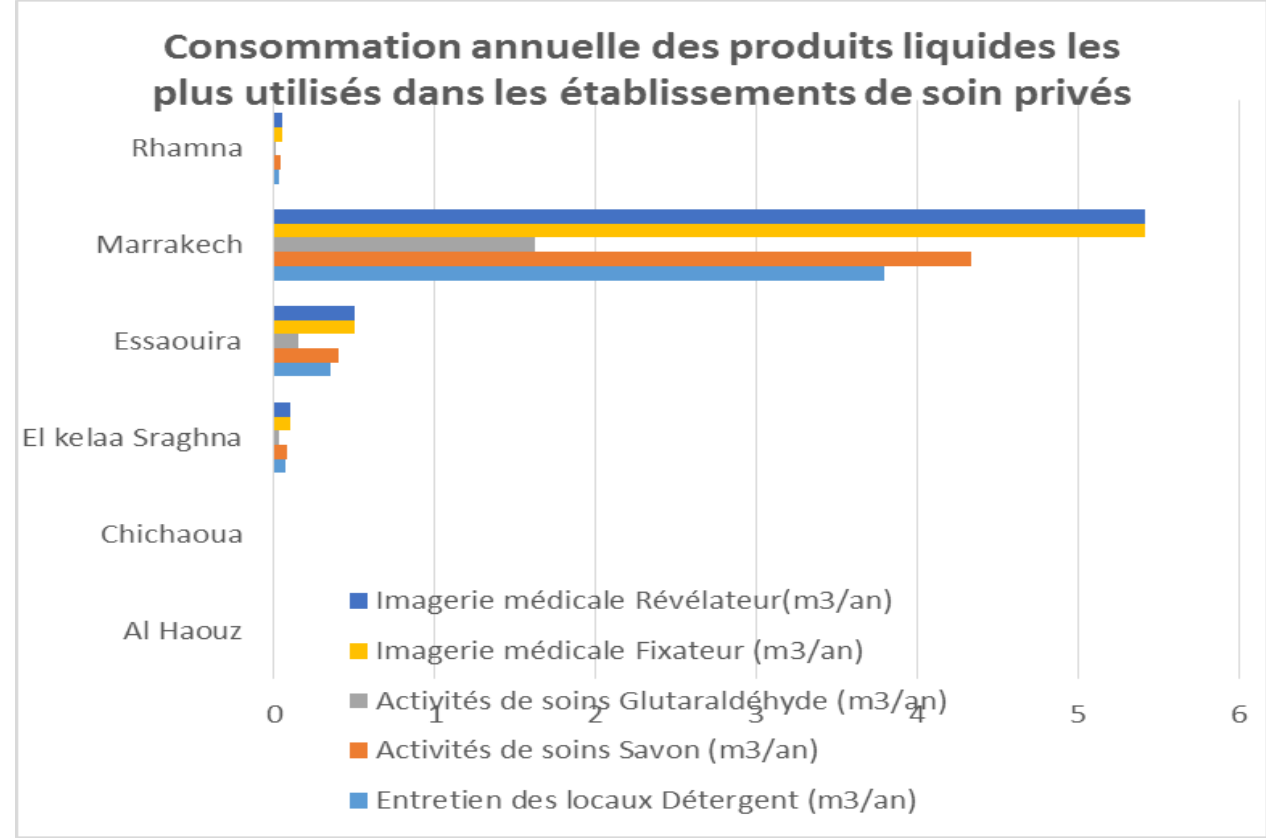

c-3 Détergent (cuisine), lessive (blanchisserie)

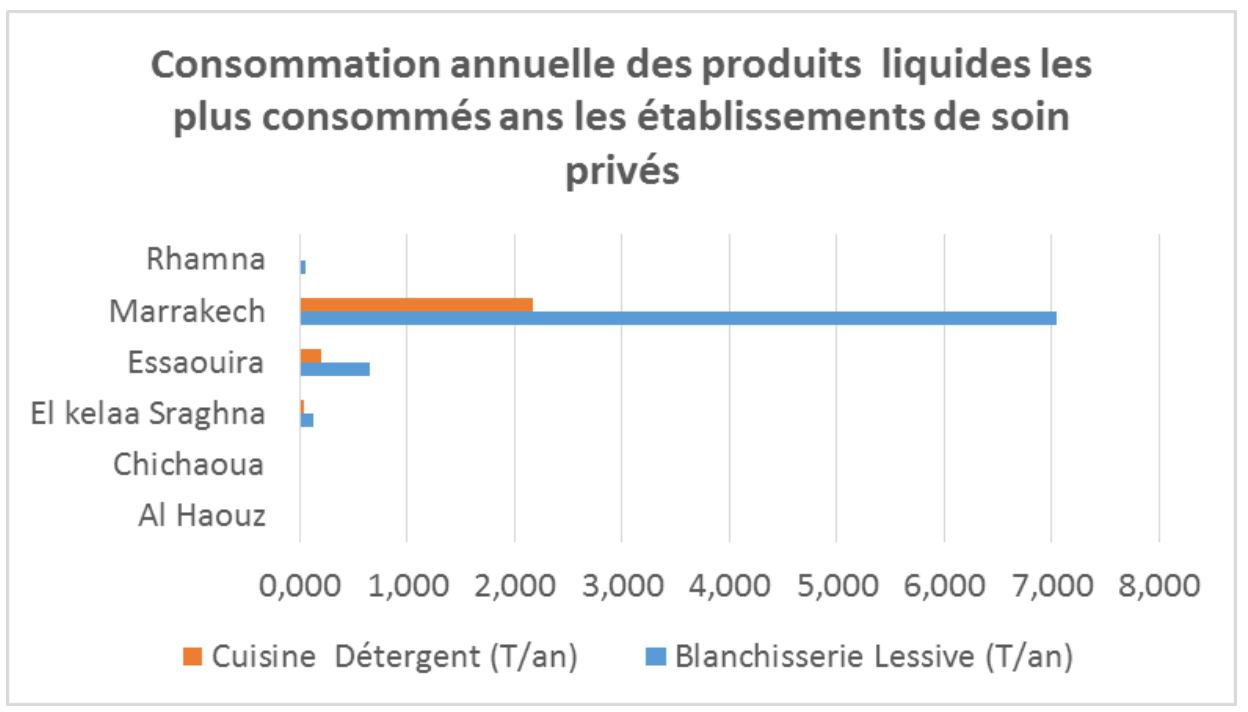

Part de contribution des rejets des établissements de soin dans la pollution communale d'une ville : Cas de la ville de Marrakech

Par sa situation géographique, la ville de Marrakech desserve toute la région de Marrakech Tensift Al-Haouz. Cette dernière s'étend sur une 
superficie de $32114 \mathrm{~km}^{2}$, soit 4,5\% du territoire national, avec une population de plus de 3,5millions que le Centre Hospitalier Universitaire Mohammed VI prend en charge.

Le tableau $n^{\circ} 2$ liste les différents établissements de soin publics au niveau de la ville de Marrakech.

Tableau 2 : Etablissements de soin publics au niveau de la ville de Marrakech, [2]

\begin{tabular}{|c|c|c|c|}
\cline { 2 - 3 } \multicolumn{2}{c|}{} & TOM & $\begin{array}{c}\text { Capacité } \\
\text { fonctionnelle }\end{array}$ \\
\hline \multirow{4}{*}{ MARRAKECH } & HGU IBN TOFEIL & 210,2 & 348 \\
\cline { 2 - 4 } & HSU ONCOLOGIE-HEMATO & 88,8 & 48 \\
\cline { 2 - 4 } & HSU IBN NAFIS & 104,9 & 211 \\
\cline { 2 - 4 } & HGR IBN ZOHR & 44,4 & 300 \\
\cline { 2 - 4 } & HSR EL ANTAKI & 37,8 & 122 \\
\cline { 2 - 4 } & HSU HOPITAL MERE ENFANT & 103,7 & 222 \\
\cline { 2 - 4 } & HSR HOPITAL PSHY.SAADA & 57,7 & 60 \\
\cline { 2 - 4 } & HSU ARRAZI SPECIALISE & 48,1 & 274 \\
\cline { 2 - 4 } & Total & 100,7 & 1585 \\
\hline
\end{tabular}

HGR : Hôpital Général Régional

HSR : Hôpital Spécialisé Régional

HSU : Hôpital Spécialisé Universitaire.

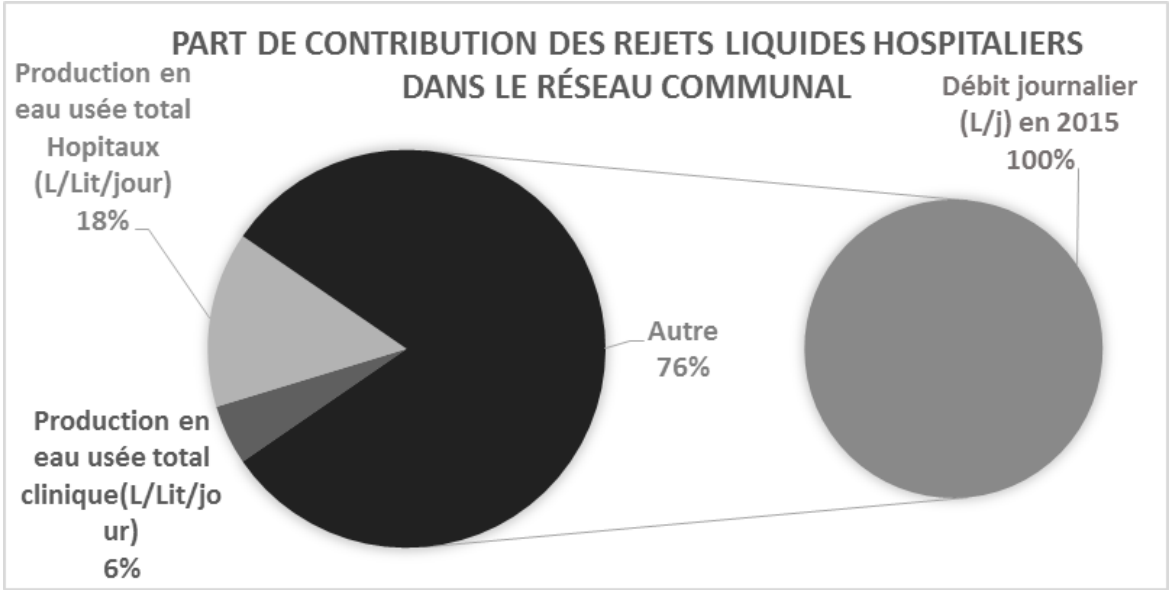

Suite aux estimations réalisées dans la présente étude, les eaux usées hospitalières issues des secteurs publics et privés présentent environ $24 \%$ du débit journalier rejeté au niveau de la ville de Marrakech. Le débit journalier des eaux usées de la ville de Marrakech en 2015 est de 143399 m3/j, selon [8]. 


\section{Pourcentage des rejets liquides hospitaliers}
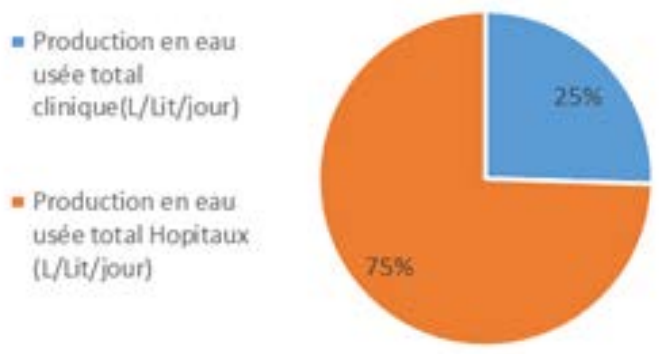

Les rejets des hôpitaux publics présentent le 3/4 et les cliniques privés le $1 / 4$ restant de l'ensemble des rejets liquides hospitaliers estimés qui rejoignent le réseau d'assainissement liquide communal au niveau de la ville de Marrakech.

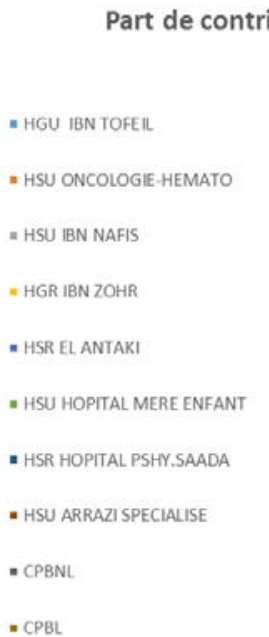

$$
\text { rejets hospitaliers }
$$

Par ailleurs, faute d'absence des données relatives aux secteurs privés diffus, les centres d'hémodialyse, les laboratoires médicaux, les facultés de médecine et de pharmacie, les rejets liquides des patients à domicile... ; ainsi que la non connaissance des pratiques de gestion de ces rejets à risque au niveau de ces organismes même à de faibles volumes, on estime que les valeurs déduites dans la présentent étude restent relativement inférieures par rapport à la fraction des rejets hospitaliers liquides réellement rejetée dans le réseau d’eau usée.

\section{Discussion}

Pour la région de Marrakech-Tensift-El Haouz, la ville de Marrakech est en tête par sa dotation en établissement de soin public et privés dans automatiquement est la plus consommatrice à l'échelle de la Région. 
Dans les pays en développement, et selon LABER et al. la consommation semble plutôt se situer autour de 500 litres par lit et par jour [17].La consommation minimale d'eau domestique est de 100 litres par habitant et par jour [18], alors que la valeur généralement admise pour les hôpitaux varie de 400 à 1200 litres par lit et par jour.

La demande moyenne en eau des établissements de santé à l'échelle internationale, en France, on estime à 750 litres par lit et par jour (soit 350 à 450 litres pour les services généraux, et 250 à 350 litres pour l'hospitalisation et la technique médicale) les besoins moyens en eau d'un Centre Hospitalier Universitaire [16]. Aux Etats-Unis d’Amérique est de 968 litres par lit et par jour [15].

Hors que pour la ville de Marrakech, on déduit un ratio d'eau potable de $200 \mathrm{~L} / \mathrm{j}$ pour le secteur privé et $180 \mathrm{~L} / \mathrm{j}$ pour le secteur public selon la capacité fonctionnelle pour l'année 2015. Des ratios très faibles pour une ville de taille par rapport à l'activité hospitalière mais ces ratios engendrent des volumes importants d'eau usée qui rejoignent le réseau d’assainissement communal. On déduit de la présente étude un pourcentage de 25\% des eaux usées hospitalières qui contiennent des substances dangereuses qui rejoignent le réseau d'eau usée au niveau de la ville de Marrakech. Sachant que les hôpitaux sont de très gros consommateurs de médicaments partiellement métabolisés, ou de désinfectants, de détergents, de solvants, ou d'autres composés, les rejets sont dirigés vers la STEP communale avec ou sans aucun traitement préliminaire. Ces eaux chargées en antibiotiques, métaux lourds, radioéléments, produits actifs et pharmaceutiques, produits d'hygiène, d'entretien, de lavage, de restauration... présentent des risques pour les espèces vivantes et le milieu récepteur surtout que même en présence de Station d'épuration, cette dernière n'est pas conçue pour traiter ce type de pollution complexe à l'échelle micrométrique.

\section{Conclusion}

L'importance de cerner la problématique des rejets liquides hospitaliers est aujourd'hui une mesure d'urgence à l'échelle internationale et c'est l'heure aujourd'hui pour qu'elle soit parmi les préoccupations stratégiques nationales au Maroc. Des mesures doivent êtres entreprises pour mieux gérer les rejets hospitaliers liquides au niveau des établissements de soin publics et privés en particulier dans les grandes villes, le cas de la ville de Rabat, Grand Casablanca, Marrakech, Fès et Tanger.

La présente étude a évalué les déchets médicaux et pharmaceutiques liquide sur le plan quantitatif qui a démontré que des volumes importants se déversent dans les réseaux d'assainissement par les établissements de soin. Ce qui impose le traitement de ces eaux usées avant leur rejet dans le réseau d'assainissement en particulier dans les grandes villes. Cependant, ces 
observations ouvrent des perspectives qui doivent être confirmées en multipliant des études locales et de réaliser d’autres analyses écotoxicologiques.

\section{References :}

1. Ulf Nielsen, «Conseil en environnement de l'eau DHI,» 2016. [En ligne].

2. M. Ministère de Santé, «SANTE EN CHIFFRES 2014, Edition 2015,»2015.[Enligne].Available:

http://www.sante.gov.ma/Publications/Etudes_enquete/Documents/0 42016/SANTE\%20EN\%20CHIFFRES\%202014\%20Edition\%20201 5.pdf. [Accès le 06-09-2016 Janvier 2016].

3. Centre de Coordination de la lutte contre les Infections Nosocomiales Interrégion Paris-Nord, «ELIMINATION DES EFFLUENTS LIQUIDES DES ETABLISSEMENTS HOSPITALIERS-Recommandations,» Institut Biomédical des Cordeliers, 15 rue de l'Ecole de Médecine- 75006 Paris, Ile de France, Picardie, Haute-Normandeie, Nord Pas-de-Calais, Décembre 1999.

4. Haut Commissariat au Plan (HCP)-Maroc, «Concepts et définitions,» Haut Commissariat au Plan (HCP)-Maroc, 2014. [En ligne]. Available: http://www.hcp.ma/glossary/Taux-d-occupationdes-lits-d-hopitaux_gw104.html. [Accès le 04 Avril 2016].

5. Ministère de la Santé, «Santé en chiffre 2012, Edition 2013,» Direction de la Planification et des Ressources financières-Division de la Plannification et des Etudes -Service des Etudes et de l'Information Sanitaire, Rabat, 2013.

6. Ministère de d'Energie, des Mines, de l'Eau et Environnement, «Schéma Directeur D'Assainissement Liquide,» Maroc.

7. Guide méthodologique : Les rejets liquides hospitaliers, Ile de France: Agence régionale de l'Energie, Conseil Scientifique de l'Envrionnement, Réseau santé qualité, Centre Antipoison Nord-Pasde-Calais, Centre Hospitalier d'Arras, Centre Hospitalier Régional Universitaire de Lille, Centre Hospitalier Roubaix, Janvier 2001.

8. BET, «Actualisation du schéma d'assainissement liquide de la ville de Mararkech (SDAL 2015),» RADEEMA, Marraekch, 2015.

9. Emmanuel. Evaluation des risques sanitaires et écotoxicologiques liés aux effluents hospitaliers,2004 INSA LYON

10. Emmanuel, Evaluation des risques sanitaires et écotoxicologiques 
liés aux effluents hospitaliers,2004 INSA LYON

11. C. DARSY, I. LESCURE, V. PAYOT et G. ROULAND, «Eflluent des établissement hospitaliers: teneur en microorganismes pathogènes, risque sanitaires, procédures particulières d'épuration et de gestion des boues,» Office International de l'Eau, LimogesFrance, Février 2002.

12. A. QADOURI, L. MOUHIR, L. SAFAADI et S. BELKADI, « Physicochemical and bacteriological pollution study of a hospital effluent with the aim of the implementation of an adequate treatment: case of ar-razi Hospital, Marrakech (Morocco).,» World journal of pharmacy and pharmaceutical sciences, vol. 04, $\mathrm{n}^{\circ}$ 11928-1947, p. 07, 2015.

13. Kümmerer, «Drugs in the environment: emission of drugs, diagnostic aids and disinfectantsinto wastewater by hospitals in relation to other sources — a review,» Chemosphere ;45:957-69, 2001.

14. K. Langford et K. Thomas , «Determination of pharmaceutical compounds in hospital effluents,» $\mathrm{n}^{\circ}$ 135:766-70, 2009.

15. . E. Emmanuel, G. Keck , J.-M. Blanchard , P. Vermande et Y. Perrodin, «Toxicological effects of disinfections using sodium hypochlorite on aquatic organisms and its contribution to AOX formation in hospital wastewater.,» Environ Int , vol. 891, n 1900, p. 30, 2004.

16. T. Ternes, «Occurrence of drugs in German sewage treatment plants and rivers.,» vol. 32, nº \%145-60, 1998.

17. T. Heberer, «Tracking persistent pharmaceutical residues in the aquatic environment: areview of recent research data.,» $n^{\circ} 1131: 5-$ 17, 2002.

18. A. Joss, E. Keller, A. Alder, A. GÖbel , C. McArdell et T. Ternes , «Removal of pharmaceuticals and fragrances in biologicalwastewater treatment,» $\mathrm{n}^{\circ}$ 139:3139-52, 2005.

19. P. Verlicci, A. Galletti, M. Pertrovic et D. Barcelo, «Hospital effluents as a source of emergingpollutants: an overview of micropollutants and sustainable treatment options,» $\mathrm{n}^{\circ} \% 1389: 416$ 28, 2010.

20. K. Brown , J. Kulis , B. Thomson , T. Chapman et D. Mawhinne, «Occurrence of antibiotics in hospital, residential, and dairy effluent, municipal wastewater, and the Rio Grande in New Mexico.,» $\mathrm{n}^{\circ}$ 1366:772-83, 2006. 
21. K. Langford et . K. Thomas, «Determination of pharmaceutical compounds in hospital effluents and their contribution to wastewater treatment works.,» Environ Int, vol. 766, n 170, p. 35, 2009.

22. L. Santos , A. Araújo , A. Fachini , A. Pena , C. Delerue-Matos et M. Montenegro , «Ecotoxicological aspects related to the presence of pharmaceuticals in the aquatic environment.,» $n^{\circ}$ 1175:45-95, 2010.

23. P. Vazquez-Roig , V. Andreu, C. Blasco et Y. Picó , «Risk assessment on the presence of pharmaceuticalsin sediments, soils and waters of the Pego-Oliva Marshlands (Valencia, eastern Spain),» $n^{\circ}$ 1440:24-32, 2012.

24. P. Hartemann, A. Hautmaniere et M. Joyeux, «La problématique des effluents hospitaliers,» vol. 13, $\mathrm{n}^{\circ} 15,2005$.

25. J. Rodier, L'analyse de l'eau : eaux naturelles, eaux residuaires, eau de mer., vol. 8e edition, Paris: DUNOD, 1996.

26. M. d. L. d. Maroc, «« Normes marocaines, Bulletin officiel du Maroc »,» Rabat, 2002.

27. E. Tahiri, L. Benaabidat, C. Nejjari et K. Benbhrahim, «Caractérisation des effluents hospitaliers de l'hôpital Al Ghassani, CHU Hassan II de Fès, Maroc,» Revue Hospitalière , vol. 47, $\mathrm{n}^{\circ} 1 \mathrm{TH} 714,2009$.

28. H. El Halouni, «Réutilisation des eaux usées en agriculture et leur impact sur l'environnement: cas de la ville d'Oujda.,» Univérsité Mohammed 1 er, Oujda, Maroc, 1995.

29. Y. El Gouamri et D. Belghyti, «Etude de la qualité physicochimique des eaux usées brutes de al ville de Saknia rejetées dans le lac Fouarat,» Journal Africain des Sciences de l'environnement, $\mathrm{n}^{\circ} 11$, pp. 53-60, 2006.

30. ONEP et GTZ, «Approche de la typologie des eaux usées urbaines au Maroc,» Rabat, 1998.

31. J. Rodier, L’analyse de l'eau naturelle, eaux résiduaires, eau de mer., 8 éme éd. Denod.

32. M. Henze, P. Harremoes , J. Jansen , C. L et E. Avrin, Wastewater treatment., Berlin: 2nd ed, 1997.

33. K. Udert, T. Larsen, A. Biébow et W. Gujer, «Urea hydrolysis and precipitation dynamics in a urine-collecting system,» Water Res, $n^{\circ}$ 137, pp. 2571-2582, 2003.

34. S. Bonté, M. Pons, O. Potier et P. Rocklin, «"Relation between Conductivity and Ion Content in Urban Wastewater",» Journal of Water Science, vol. 21, n 14, pp. 429-438, 2008. 
35. M. Benykhlef, S. Naji, D. Belghyti et Y. El Guamri , «CARACTERISATION PHYSICO-CHIMIQUE DES EAUX USEES INDUSTRIELLES DU GRAND AGADIR (MAROC) : CAS D’UNE HUILERIE,» vol. 3, nº 1N 110707, 2011.

36. P. Du Chaufour, «Abrégé de pédologie: sol, végétation et environnement,» Masson, 5è édition , 1997.

37. N. Ameziane et L. Benaabidate, «Caractérisation microbiologique des effluents de l'hôpital Mohamed V de Meknès et étude de leur impact sur l'environnement,» 2013.

38. M. Baudu, C. Dagot, D. Chainier, M.-C. Ploy, L. Foan et M. Rabiet, «Identification d'une signature biologique et physico-chimique des effluents hospitalier : traçage dans l'environnement,» $\mathrm{n}^{\circ} \% 130,2009$.

39. Emmanuel, Evaluation des risques sanitaires et écotoxicologiques liés aux effluents hospitaliers, vol. 259p, Lyon: Thèse INSA de Lyon, Spécialité Sciences et Techniques du Déchets, 2004.

40. E. Emmanuel, Y. Prrodin, G. Keck, J. Blanchard et P. Vermande, «Ecotoxicological risk assessment of hospital wastewater : a proposed framwork for raw efflents discharging into urban swer network,» vol. 117, n 11, 2005.

41. D. Zmirou, J. Ferley, J. Collin, M. Charrel et J. Berlin, «A follow-up study of gastro-intestinal diseases related to bacteriologically substandard drinking water,» American Journal of Public Health, vol. 77, $\mathrm{n}^{\circ}$ 582-584, 1987.

42. R. El ALAOUI et . I. TAOUSSI, «L'impact du Chrome VI sur le traitement des eaux usées dans la station d'épuration de Marrakech,» Université Cadi Ayyad -Faculté des Sciences et TechniquesLaboratoire Géoressources-Unité de Recherche Associée $\mathrm{Au}$ CNRST (URAC 42), Marrakech, 2013.

43. Kümmerer et Helmers, «Hospital effluents as a source for platinum in the environment:179-84,» Sci Total Environ, 1997.

44. Metcalf et Eddy, Wastewater engineering: Traetment, Disposal and Reuse, 3ème Edition Library of Congress Cataloging in publication data. TD. 645.T34, 1991.

45. R. Hamdaoui, «Caractérisation physico-chimique et traitement des eaux usées résiduaires de la société SETEXAME,» Mémoire DESA Fac.Sci, Kénitra, 2006.

46. F. Orias et P. Yves, "Characterisation of ecotoxicity of hospital effluents:A review,» Science of the Total Environment, pp. 454-455, 2013. 
47. C. Boillot, C. Bazin, F. Tissot-Guerraz, J. Droguet, M. Perraud, J. Cetrec et et al., «Daily physicochemical,microbiological and ecotoxicological fluctuations of a hospital effluent,» $n^{\circ} 1403: 113$ 29, 2008.

48. B. Halling-Sørensen , S. Nors Nielsen , P. Lanzky , F. Ingerslev, H. Holten Lützhøft et S. Jørgensen, «Occurrence, fate and effects of pharmaceutical substances in the environment - a review,» $n^{\circ}$ 136:357-93., 1998.

49. . P. Jjemba, «Excretion and ecotoxicity of pharmaceutical and personal care products in the environment.,» $n^{\circ}$ 163:113-30., 2006.

50. J.-P. Besse et J. Garric , «Human pharmaceuticals in surface waters: implementation of a prioritization methodology and application to the French situation.,» $\mathrm{n}^{\circ}$ 1176:104-23., 2008.

51. A. Kumar et I. Xagoraraki, «Pharmaceuticals, personal care products and endocrine-disrupting chemicals in U.S. surface and finished drinking waters: a proposed ranking system.,» n \%1408:5972-89, 2010.

52. Q. Sui , B. Wang , W. Zhao , J. Huang , G. Yu et S. Deng , «Identification of priority pharmaceuticals in the water environment of China,» $\mathrm{n}^{\circ}$ 189:280-6, 2012.

53. O. Thomas, «" Métrologie des eaux résiduaire ».»"Ed. Cebedoc / Tec. et Doc. 11 Liège -75384. Paris, 1985.

54. O. Thoma, Métrologie des eaux résiduaire., 1995: Ed. Cebedoc / Tec.et Doc. 11, Liège -75384. Paris..

55. R. H. S. R. LABER J., «Two-stage constructed wetland for treating hospital wastewater in Nepal.,» Wat. Sci. Tech, vol. 40, n%13, pp. 317-324, 1999.

56. G. F, Le monde manquera-t-il bientôt d’eau? Sécheresse, vol. 6, 1:11-15, 1995.

57. I. B. d. Cordeliers, «CLIN (Coordination de Luttes contre les Infections Nosocomiales), Élimination des effluents liquides des établissements hospitaliers - Recommandations.,» Institut Biomédical des Cordeliers, Paris Nord, 1999, p. 74..

58. USEPA, «Preliminary data summary for the hospitals point source category. Office of water regulations,» Office of water, United States Environmental Protection Agency, , Washington, 1989a, EPA 440/189/060-n, 76 p. 